\title{
Neural network prediction of geomagnetic activity: a method using local Hölder exponents
}

\author{
Z. Vörös and D. Jankovičová \\ Geomagnetic Observatory Hurbanovo, Geophysical Institute SAS, Slovak Republic \\ Received: 14 December 2001 - Revised: 19 February 2002 - Accepted: 3 March 2002
}

\begin{abstract}
Local scaling and singularity properties of solar wind and geomagnetic time series were analysed using Hölder exponents $\alpha$. It was shown that in analysed cases due to the multifractality of fluctuations, $\alpha$ changes from point to point. We argued there exists a peculiar interplay between regularity/irregularity and amplitude characteristics of fluctuations which could be exploited for the improvement of predictions of geomagnetic activity. To this end, a layered back-propagation artificial neural network model with feedback connection was used for the study of the solar wind magnetosphere coupling and prediction of the geomagnetic $D_{s t}$ index. The solar wind input was taken from the principal component analysis of the interplanetary magnetic field, proton density and bulk velocity. Superior network performance was achieved in cases when the information on local Hölder exponents was added to the input layer.
\end{abstract}

\section{Introduction}

One of the goals of solar-terrestrial physics is to predict the response of the magnetosphere-ionosphere system to highly variable conditions in the solar wind (SW). The question of solar wind-magnetosphere coupling (SWMC) can be studied by means of input-output modelling. Linear inputoutput techniques (or linear prediction filtering) describe the SWMC by a linear moving-average (MA) filter, assuming that the convolution of a time-invariant transfer function (TF), with an earlier SW input, can predict the magnetospheric output represented by time series of geomagnetic indices (Iyemori et al., 1979; Bargatze et al., 1985; McPherron et al., 1988). The TF characterizes the magnetospheric response and can be estimated directly from data provided that a sufficiently large number of input-output pairs is available. In fact, Bargatze et al. (1985), using the $v B_{z}-A L$ inputoutput data $\left(v\right.$ - solar wind velocity, $B_{z}$ - interplanetary magnetic field $N-S$ component, $A L$ - auroral zone geomagnetic

Correspondence to: Z. Vörös (geomag@geomag.sk) index), have shown that the linear MA filters can identify two different regimes in which SW energy is dissipated within the magnetosphere (directly driven and loading-unloading regimes). At the same time, the best linear MA filters do not predict the geomagnetic output precisely, unless strongly varying filter parameters are considered in each case of activity level separately (Blanchard and McPherron, 1994). Different levels of geomagnetic activity and the nonlinearity of the SWMC were then treated by nonlinear MA filters (Price et al., 1994; Vassiliadis et al., 1995) using the assumption that the geomagnetic activity is a nonlinear function of the solar wind input. Actually, local linear (i.e. nonlinear) MA filters were used, which represent a linear approximation of the nonlinear system. Nonlinear MA filters proved to be better predictors of geomagnetic response than the linear ones, but the internal dynamics of the magnetosphere and the additional influence of it on the geomagnetic response itself (a feedback) was more explicitly considered within the frame of state-input space models (Vassiliadis et al., 1995). Here the prediction of magnetospheric states is made within a common input (solar wind) output (geomagnetic data) phase space, and the local linear (nonlinear) approximation is given by an evolution of the nearest neighbours of a phase space point. Vassiliadis et al. (1995) found that in comparison with linear state-input models (global aproximation), the nonlinear state-input models (local approximation based on nearest neighbours) give better predictions of geomagnetic activity.

An alternative to the above MA filters is represented by artificial neural networks (ANN) which are global nonlinear functions. Elman recurrent ANN was used by Munsami (2000) to model the SW forcing of the westward auroral electroject and the storm-time ring current. In predicting geomagnetic activity, their performance was similar to that of linear filters (Hernandez et al., 1993). Significantly better performance was achieved by gated ANNs that accounted for different levels of activity. Weigel et al. (1999) used three individual ANNs for modelling low, medium and high $v B_{z}, A L$ activity levels using data from the database of Bargatze et al. (1985). The outputs of these ANNs, to- 
14 January 1998, 05:20--10:20 UT
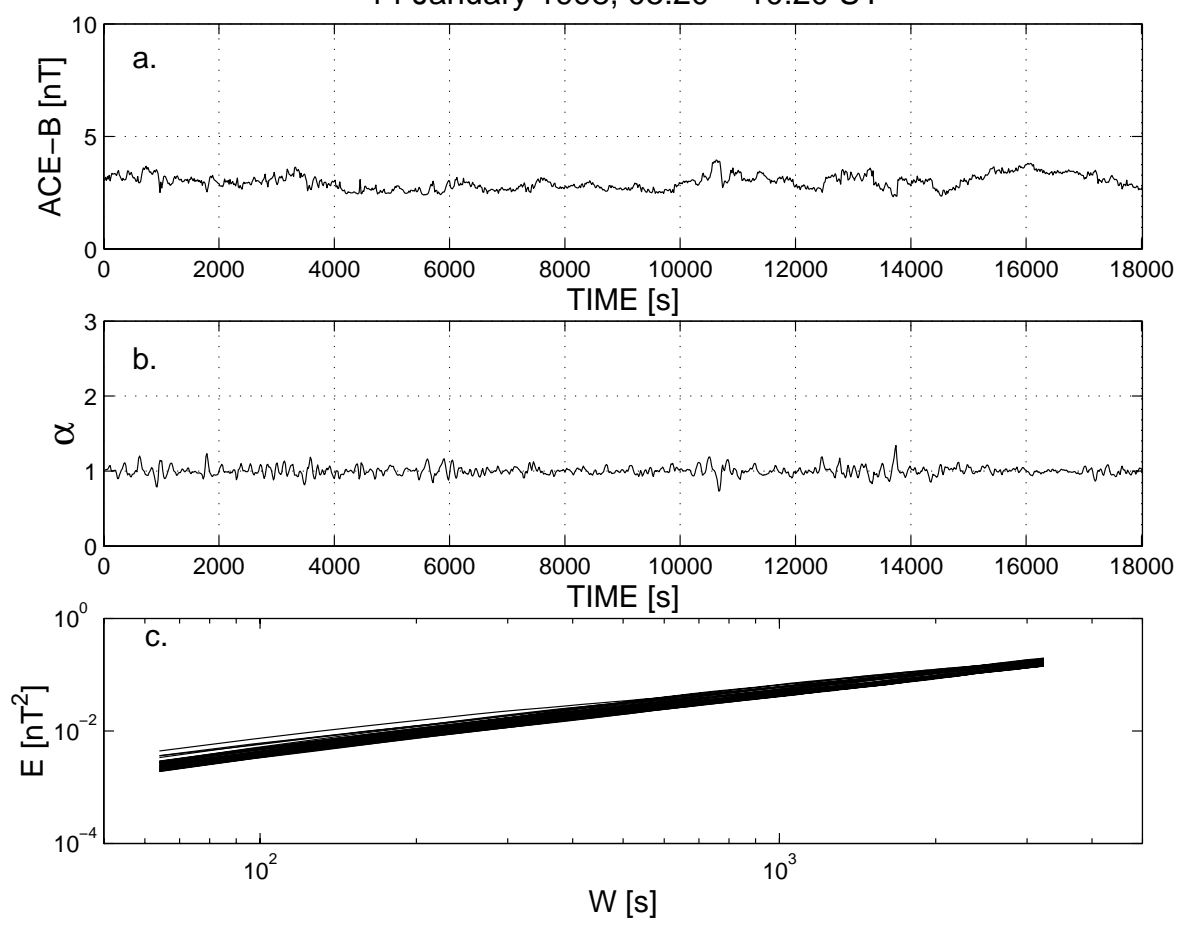

Fig. 1. Period of low activity level; (a) Interplanetary magnetic field from ACE satellite (time resolution 16 [s]); (b) The estimated time series of Hölder exponents $\alpha$ (c) The energy content of the signal versus window length $W$.

gether with past geomagnetic outputs, were used to train the gate network. It was shown by Weigel et al. (1999) and Weigel (2000) that the gated architecture gave significantly better predictions than the ungated one or the ARMA system reported by Hernandez et al. (1993). Obviously, the gated ANN architecture resembles the state-input space model of Vassiliadis et al. (1995), accounting for changing activity levels. Local linear filters can be calculated in a neighbour of any point in state-input space; the gated ANN, however, uses only three levels of activity.

In this paper, we propose a method which allows one to consider the changing level of SW fluctuations. Instead of building a more structured gated ANN architecture, we use the extra information on local scaling characteristics of properly introduced measures which can be estimated directly from a time series. Multifractals exhibit time-dependent scaling laws and hence, allow for a description of irregular phenomena that are localized in time. Multifractal scaling characteristics of geomagnetic fluctuations were studied by Consolini et al. (1996) and Vörös (2000). Jankovičová et al. (2001), using multilayer feed-forward ANNs, have shown that the information on multifractal characteristics of geomagnetic data added to the input enhanced the performance of their ANN in reconstructing AE-index time series from geomagnetic observatory data. The inclusion of multifractality, however, somewhat amplified the noise component in this case. We expect that the inclusion of the scaling characteristics of solar wind and geomagnetic fluctuations to the ANN modelling of SWMC offers a way for considering essential local information on rapid changes, irregularities and intermittence not considered enough hitherto. Intermittence of SW and geomagnetic fluctuations were not built into the nonlinear filter or ANN models. Notwithstanding that SW fluctuations proved to be strongly intermittent (Burlaga, 1991; Carbone, 1994; Marsch et al., 1996; Tu et al., 1996; Bruno et al., 1999) and also both nonlinear magnetotail theories (Chang, 1999; Chapman et al., 1998; Klimas et al., 2000) and experimental works (Consolini et al., 1996; Borovsky et al., 1997; Consolini and De Michelis, 1998; Consolini and Lui, 1999; Vörös, 2000; Kovács et al., 2001; Watkins et al., 2001) predict or confirm the presence of scalings, multifractality and intermittence within the magnetosphere. Though there exist competing theoretical concepts regarding the underlying physical mechanisms which may or may not produce the observed scalings (Freeman et al., 2000; Antoni et al., 2001), these considerations have no effect on our analysis. We simply ask what are the scaling characteristics of fluctuations and how can this information improve our ability to predict geomagnetic activity using ANNs?

\section{Data analysis methods}

\subsection{Local scaling characteristics: the Hölder exponents}

We consider the accumulated amount of signal energy within a window $W:\left(t_{i}-W, t_{i}\right)$. The signal energy $E$ within a 

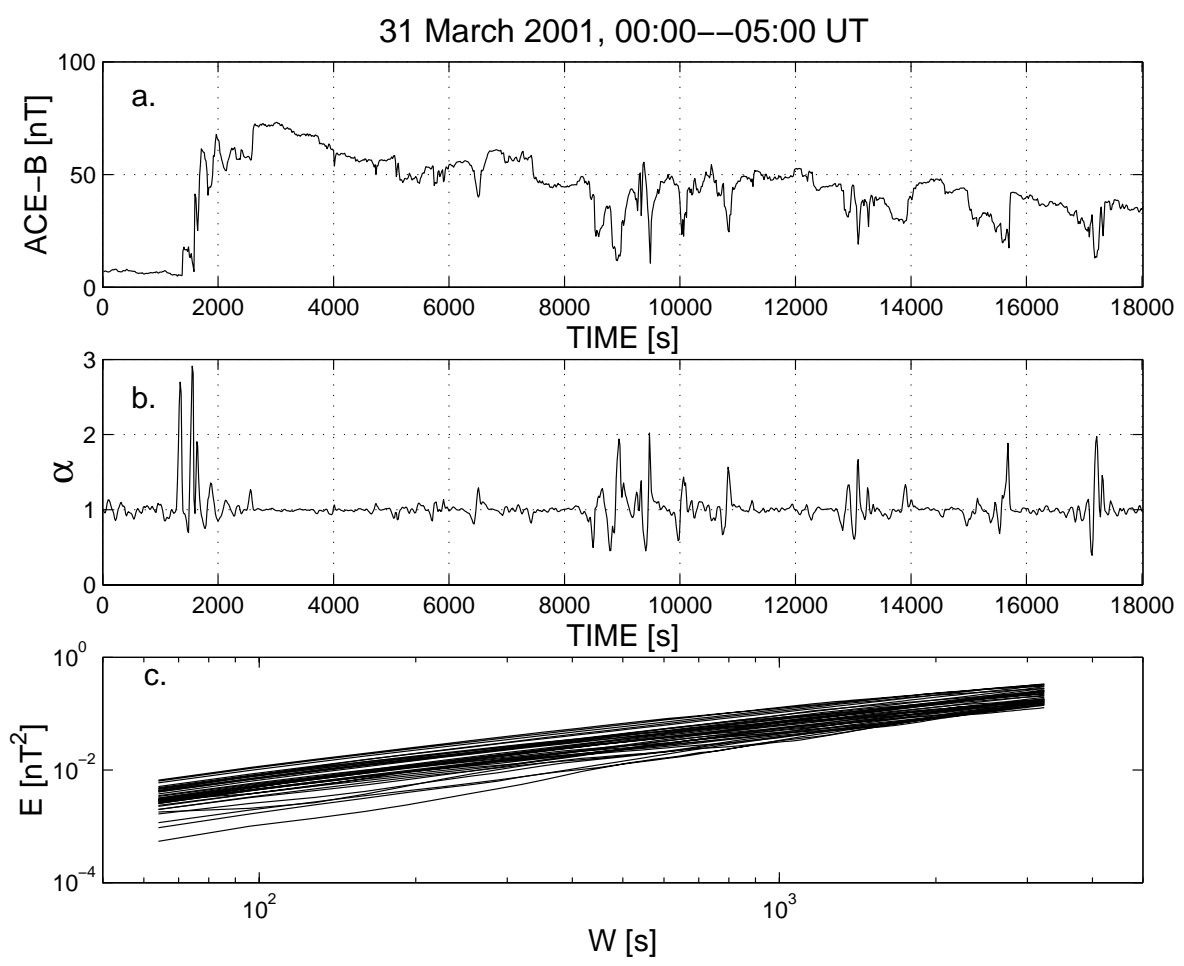

Fig. 2. Period of high activity level; - same as in Fig. 1.

window $W$ is computed as a sum of the squared amplitudes of time series through

$E\left(t_{i}\right)=\sum_{i-W}^{i} X^{2}\left(t_{i}\right) ; \quad i=1,2, \ldots, N$

and

$\sum_{i=1}^{N} X^{2}\left(t_{i}\right)=1$,

where $X\left(t_{i}\right)$ represents a time series, $N$ is the total number of data points. The distribution of $E$ in time is considered to be a measure which may also appear as singular. Mathematically, a measure can be characterized by its density. An erratic behaviour appears in the absence of a density for a singular measure. Generally, singular distributions can be characterized locally by the so-called singularity or Hölder exponents $\alpha$ (Halsey et al., 1986; Muzy et al., 1994; Véhel and Vojak, 1998). Loosely speaking, the exponent $\alpha$ quantifies the degree of regularity or irregularity (singularity) in a distribution or a function in a point $t_{i}$. Usually, the measure $E\left(t_{i}, W\right)$ within a window $W$ scales as $W^{\alpha}$. Therefore, $\alpha$ can be estimated by a regression method using

$\alpha\left(t_{i}, W\right)=\frac{\log E\left(t_{i}, W\right)}{\log W}$

taking different window lengths $W$. For a monofractal $\alpha\left(t_{i}\right)=$ const for all $t_{i}$, while in a case of multifractal measure (non-uniform distribution), $\alpha$ changes from point to point (non-stationarity). For instance, fractional Brownian motion or continuous Itô processes represent self-affine fluctuations governed by a single Hölder exponent. The global distribution of singularity exponents $\alpha$ for geomagnetic fluctuations was studied by Consolini et al. (1996) and Vörös (2000). It was shown that on the time scale of substorms and storms, geomagnetic fluctuations seem to be analogous to the simple multiplicative $p$-model, which describes energy cascade processes in turbulent flows. This model explains how a specific energy flux introduced on large scales to a flow can lead to non-homogeneous, intermittent energy distributions on small scales. On this basis, we expect that in the case of a homogeneous energy transfer rate between scales with no intermittency effects, the above defined distribution will be stationary and $\alpha\left(t_{i}\right) \sim 1$ for all $t_{i}$. Otherwise, $\alpha\left(t_{i}\right)<1$ indicate irregularities, sharp variations around $t_{i}$, while $\alpha\left(t_{i}\right)>1$ is found in regions where events are more regular (Riedi and Véhel, 1997). In the case of multifractal processes, $\alpha$ changes from point to point, which usually makes the numerical estimation of $\alpha$ s difficult. A number of papers deals with this question (Muzy et al., 1994; Jaffard and Meyer, 1996; Mallat and Hwang, 1992; Véhel and Vojak, 1998). Though the Hölder exponents do not characterize the local regularity properties of a signal completely (Guiheneuf et al., 1998), we are going to use the simple relation (3) to show that even a rough estimation of local scaling characteristics of the signal may enhance the performance of ANNs. We note that a running numerical estimate of $\alpha$ may fluctuate sharply for other, yet multifractality different, nonstationary processes. 

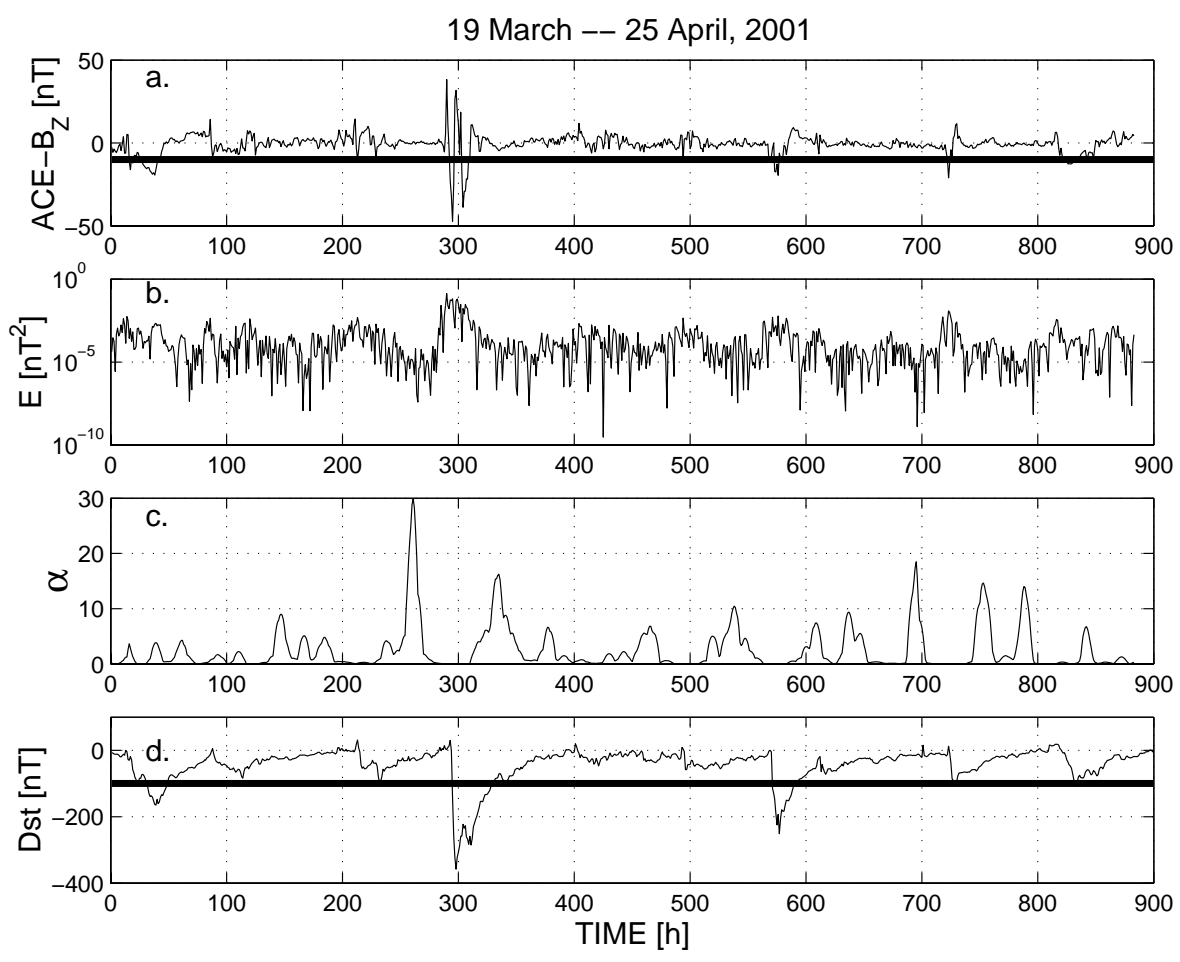

Fig. 3. (a) Interplanetary magnetic field $B_{z}$ component (time resolution $1 \mathrm{~h}$. (b) The corresponding energy content $E$. (c) The Hölder exponents. (d) Geomagnetic $D_{s t}$ index.

\subsection{ANN description}

A layered back-propagation ANN model (Rumelhart et al., 1986; Kröse and Smagt, 1996) with feedback connection from output layer to input layer was constructed. The outputinput layer connection makes the output history to be an ordinary input unit in the training process. The output of the model can be expressed in the form

$$
\begin{aligned}
y(t & +\Delta \tau)=F\left(\sum _ { k = 1 } ^ { Q } w _ { k } f _ { k } \left(\sum_{j=0}^{T} v_{j k} I_{j}^{(1)}(t-j \Delta \tau)\right.\right. \\
& +\sum_{j=0}^{T} v_{j k} I_{j}^{(2)}(t-j \Delta \tau) \\
& \left.\left.+\sum_{i=0}^{T} u_{i k} y(t-i \Delta \tau)+v_{0}\right)+w_{0}\right)
\end{aligned}
$$

where $y$ denotes the $D_{s t}$ time series; the two inputs equal $I^{(1)} \equiv P c 1$ and $I^{(2)} \equiv P c 2 ; T$ the history; $\Delta \tau$ the time resolution $(\Delta \tau=1 \mathrm{~h}) ; u_{i k}, v_{j k}$ the weights between input and hidden layers; $w_{k}$ the weights between hidden and output layers; $v_{0}, w_{0}$ the biases of the layers; $Q$ the number of hidden units; $F$ and $f_{k}$ the nonlinear activation function. In our model, $f_{k}$ is the hyperbolic tangent, $F$ represents the linear activation functions and $Q=6$. The performance of the ANN model was evaluated through root mean-squared error
(RMSE) and correlation coefficient $(\rho)$

$$
\begin{gathered}
R M S E=\sqrt{\left(\left(\sum_{i=1}^{N}\left(y_{i}^{\text {out }}-y_{i}^{\text {pred }}\right)^{2}\right) / N\right)} \\
\rho=\frac{\sum_{i=1}^{N}\left(y_{i}^{\text {out }}-\bar{y}^{\text {out }}\right)\left(y_{i}^{\text {pred }}-\bar{y}^{\text {pred }}\right)}{\sigma_{y^{\text {out }}} \sigma_{y} \text { pred }}
\end{gathered}
$$

where $y^{\text {out }}$ denotes an actual output, $\bar{y}^{\text {out }}$ its mean value and $y^{\text {pred }}$ a one-step ahead prediction of ANN, $\bar{y}^{\text {out }}$ its mean value; $N$ is their length; $\sigma_{y}$ out and $\sigma_{y}$ pred are the standard deviations of $y^{\text {out }}$ and $y^{\text {pred }}$.

\section{Data analysis}

In this paper, we are going to predict the $D_{s t}$ index one hour in advance using the layered back-propagation ANN model with feedback connection. Prior to that, we show several examples which demonstrate that the Hölder exponents estimated by Eq. (3) provide local characteristics of the analysed time series that are sensitive enough to capture the necessary information on the abrupt changes and activity levels.

Figure 1a shows the interplanetary magnetic field (IMF) variations registered by the ACE satellite, which is continuously monitoring the $\mathrm{SW}$ at the $L_{1}$ Earth-Sun Lagrange point. The time resolution is $16 \mathrm{~s}$ and $5 \mathrm{~h}$ of data are shown from 14 January 1998, 05:20 UT. This is a time period of 

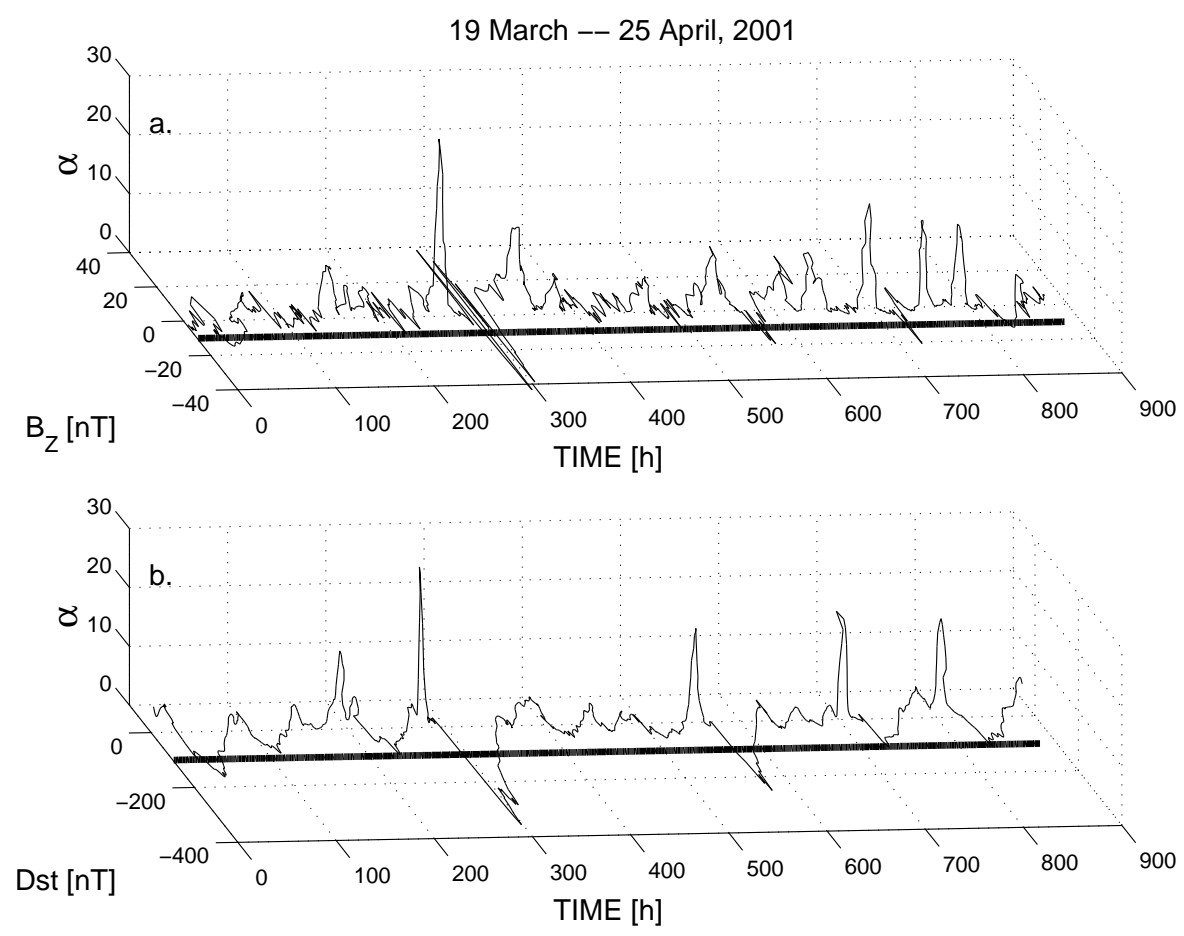

Fig. 4. The interplay between regularity/irregularity and amplitude characteristics; (a) Interplanetary magnetic field $B_{z}$. (b) Geomagnetic $D_{s t}$ index.

very low activity level with a mean value of IMF ACE $B$ fluctuations of $3 \mathrm{nT}$. The Hölder exponents estimated within the variable window length $W \in(16,16 * 160) \mathrm{s}$ at each point are depicted in Fig. 1b. It is visible that $\alpha$ fluctuates around its mean value $\bar{\alpha} \sim 1$, which means that the measure is almost uniformly distributed. The energy content of the signal $E$, and its scaling with window length, i.e. $\sim W^{\alpha}$, is shown in a log-log plot in Fig. 1c.

In contrast with Fig. 1, Fig. 2 shows a more disturbed period of IMF ACE $B$ variations from 31 March 2001 from 00:00 to 05:00 UT. The mean value of $B$ is $43 \mathrm{nT}$. Large departures from $\bar{\alpha}=1$ are present (Fig. 1b), mainly within time periods of enhanced fluctuations. These periods are characterized by a sudden increase of regularity $(\alpha>\bar{\alpha})$ followed by periods of low regularity $(\alpha<\bar{\alpha})$ or vice-versa.

In fact, $\alpha$ appears to be a sensitive indicator of fluctuations which may occur during periods of enhanced IMF $B$ amplitudes; however, when the fluctuations cease, the values of $\alpha$ return to $\bar{\alpha} \sim 1$, independent of the actual amplitudes. A good example of it is visible within the time interval $t \in(2600,5000) \mathrm{s}$ in Figs. $2 \mathrm{a}$ and $2 \mathrm{~b}$, where $B>50 \mathrm{nT}$ and $\alpha \sim 1$. Moreover, the local fluctuations of $\alpha$ around $\bar{\alpha}$ seem to be larger when the gradient of $B$ increases, but it is not always valid (not shown). There is also a clear difference between the scalings in Figs. 1c and 2c.

We conclude that, besides the amplitude of magnetic field variations, the local scaling properties of the signal described by Hölder exponents $\alpha$ (Eq. 3) may represent an essential piece of information, the consideration of which would allow for a better prediction of future geomagnetic activity.

Other examples of longer period data sets (from 19 March to 25 April 2001) are depicted in Fig. 3. This time, IMF $B_{z}$ from the ACE satellite and the $D_{s t}$ index are considered with a time resolution of $1 \mathrm{~h}$. The thick line in Fig. 3a corresponding to $B_{z}=-10 \mathrm{nT}$ highlights periods of enhanced SWMC. Gonzalez and Tsurutani (1987) have shown that the interplanetary causes of intense magnetic storms $\left(D_{s t}<-100\right.$ $\mathrm{nT})$ are long in duration $(>3 \mathrm{~h})$ large and negative $(<-10$ nT) $B_{z}$ events associated with interplanetary duskward electric fields $>5\left[\mathrm{mVm}^{-1}\right]$. A comparison of Figs. 3a and 3d shows an agreement with the above criteria, i.e., long in duration, negative IMF $B_{z}$ events occur, together with intense magnetic storms. The horizontal thick line corresponds to the limit of $D_{s t}=-100 \mathrm{nT}$ in Fig. 3d. Figure 3b shows the normalized measure $E$ and the estimated Hölder exponents are in Fig. 3c. Approximately the same behaviour is visible as previously (Fig. 2), which may be even better visualised by drawing 3D plots of time, IMF $B_{z}$ or $D_{s t}$ index and the corresponding Hölder exponents, as in Figs. $4 \mathrm{a}$ and $4 \mathrm{~b}$. In both cases, when the above mentioned physical limits of amplitudes $\left(B_{z}<-10 \mathrm{nT}\right.$ and $\left.D_{s t}<-100 \mathrm{nT}\right)$ are crossed, the Hölder exponents have their local minima, $\alpha<\bar{\alpha}$, indicating sharp irregular variations. Intense magnetic storms $\left(D_{s t} \leq-100 \mathrm{nT}\right.$ and $\left.\alpha<\bar{\alpha}\right)$ are usually preceeded by sudden increases of $\alpha \gg \bar{\alpha}$, i.e. by short periods of increased regularity (Fig. 4b). The same effect is present in the $B_{z}$ time series (Fig. 4a), though, except for the large event around $\sim 300 \mathrm{~h}$, it is less visible. 
$\mathrm{D}_{\text {st }}$ index from 1 January to 28 June 2001

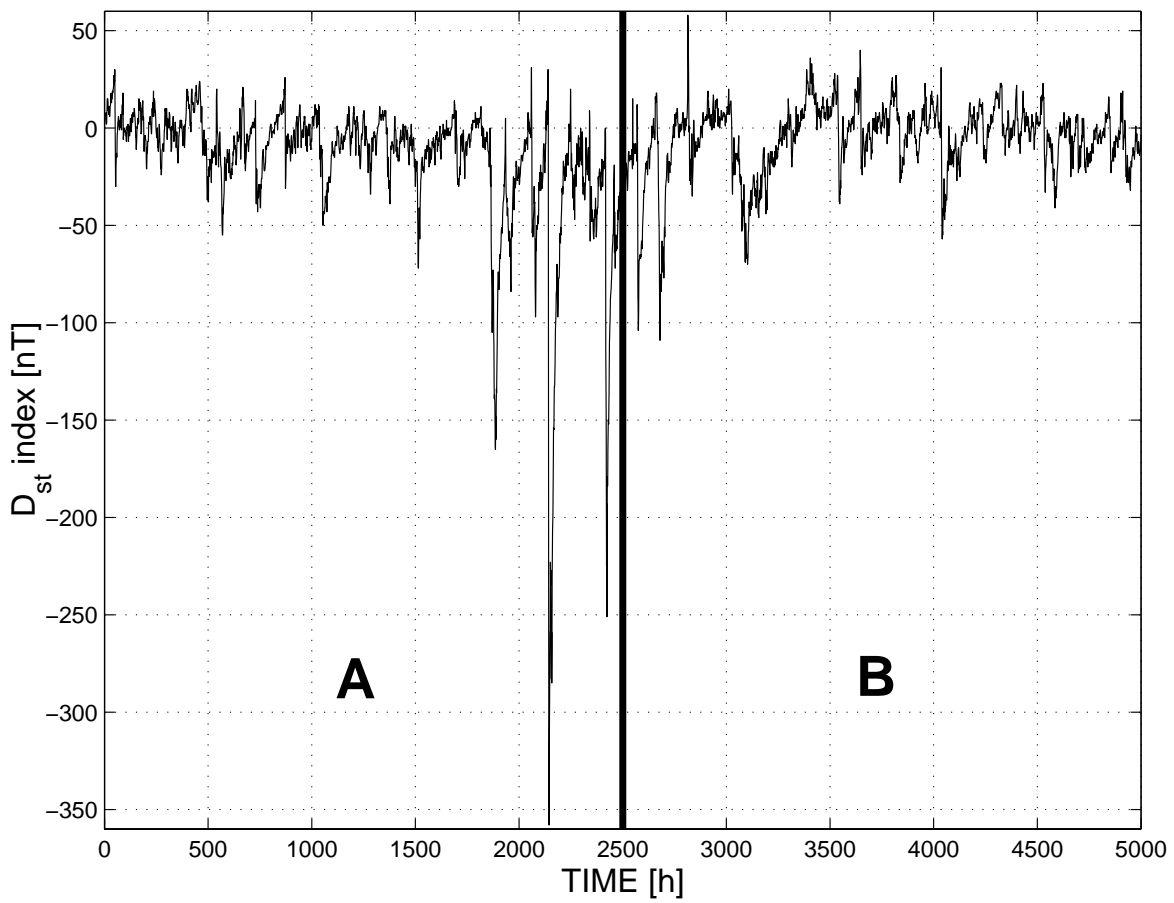

Fig. 5. $D_{\text {st }}$ time series from 1 January 2001 to 28 July 2001 used for ANN analysis ((a) The period for training process. (b) Independent set for prediction; thick vertical line divides (a) and (b)).

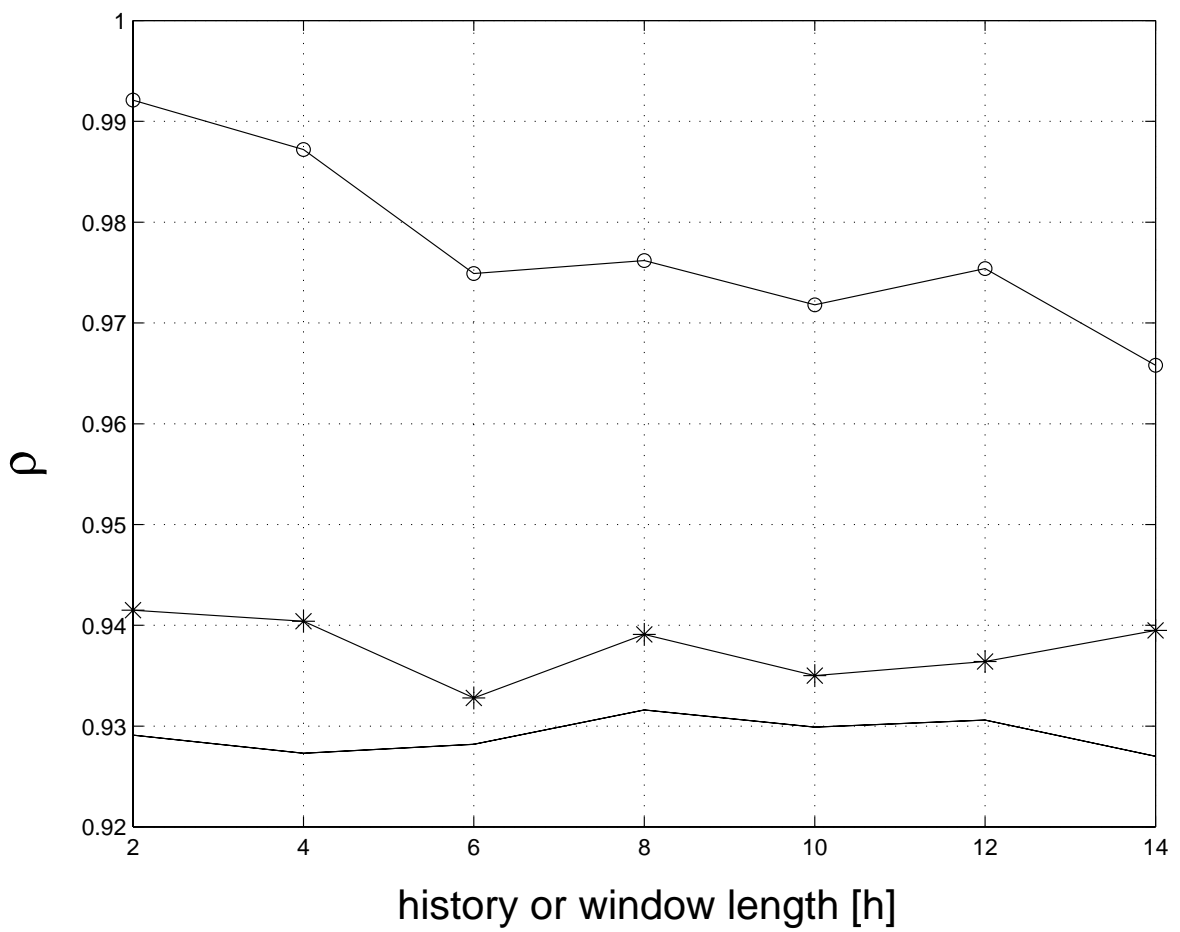

Fig. 6. Correlation coefficient dependence on history $T$ or window length $W(T=W)$; - - without Hölder exponents; * - Hölder exponents for vectors of $P c 1$ and $P c 2$ inputs considered; o - Hölder exponents for inputs $P c 1$ and $P c 2$ and for past $D_{s t}$ index considered. 

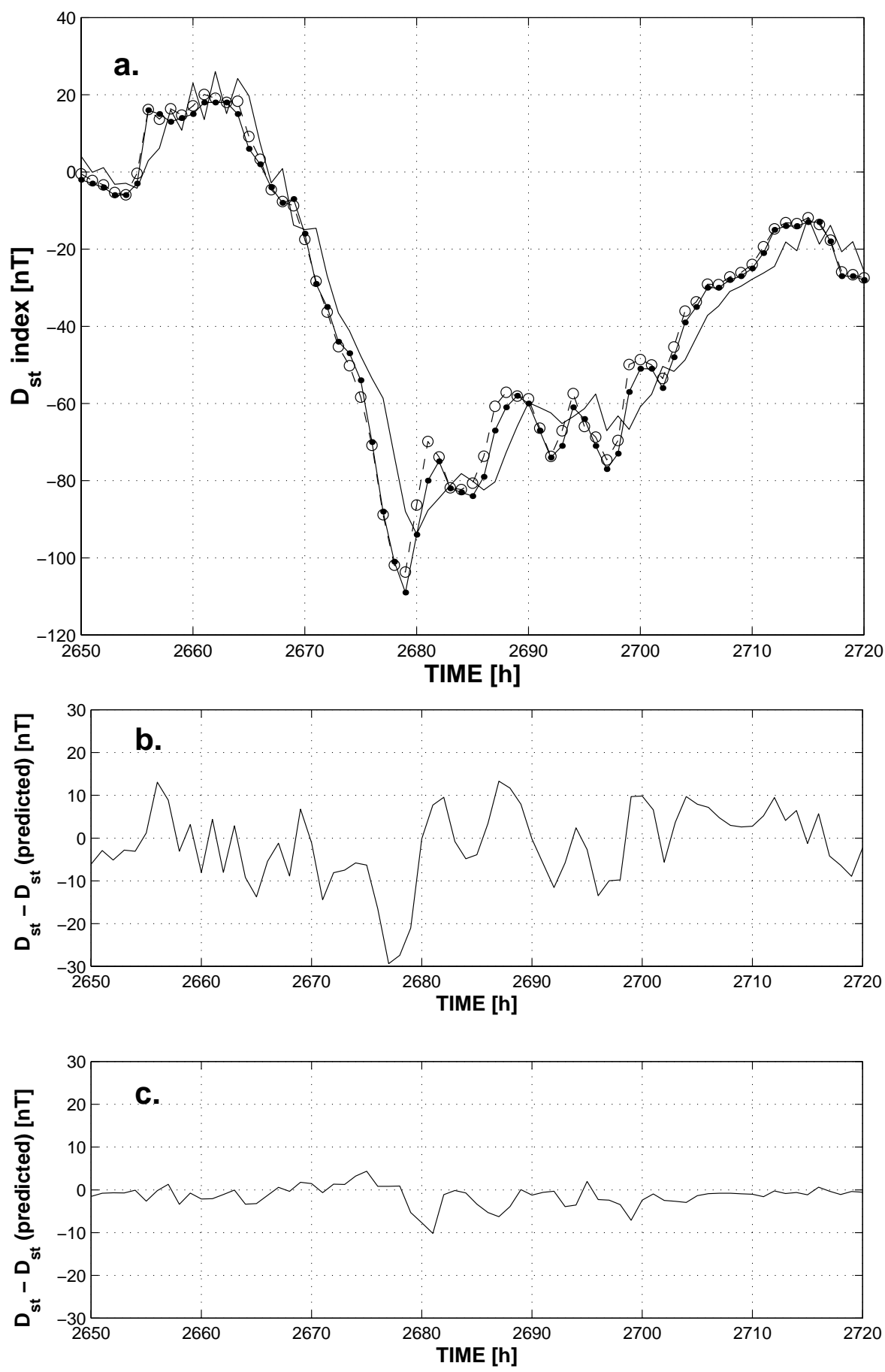

Fig. 7. (a) $1 \mathrm{~h}$ ahead prediction of $D_{s t}$ index for period from 10 March 2001, 10:00 UT to 13 March 2001, 08:00 UT (• - actual output; - prediction without Hölder exponents; o - prediction with Hölder exponents of $P c 1, P c 2$ and $D_{s t}$ on input. (b) Differences between actual $D_{s t}$ and predicted $D_{s t}$ without Hölder exponent time series. (c) Differences between actual $D_{s t}$ and predicted $D_{s t}$ with Hölder exponent time series on input.

We expect that precisely the interplay between regularity/irregularity and amplitude characteristics should be understood from ANNs to achieve superior performance. The simplest way to realize this is to add, besides the amplitudes of the analysed variables, the corresponding series of Hölder exponents to the ANN input. The following ACE SW pa- rameters with $\Delta \tau=1 \mathrm{~h}$ time resolution were used: $B_{x}, B_{y}$, $B_{z},|\mathbf{B}|, n, v$. The time evolution of $1 \mathrm{~h} D_{s t}$ index from 1 January to 28 July 2001 was considered. The time series of SW parameters were preprocessed using principal component $(P c)$ analysis (Gnanadesikan, 1977; Reyment and Jöreskog, 1996). The linear combinations of normalized SW 
parameters, their derivatives and combinations: $B_{x}, B_{y}, B_{z}$, $|\mathbf{B}|, n, v, n v, n|\mathbf{B}|, v|\mathbf{B}|, v n|\mathbf{B}|, d B_{x} / d t, d B_{y} / d t, d B_{z} / d t$, $d|\mathbf{B}| / \mathbf{d t}, d v / d t, d n / d t$ were used for the calculation of the $P c$ s. It was shown by Jankovičová et al. (2002) that, for the considered set of SW parameters, most of the variance of SW fluctuations is controlled by the first two components. In this paper, we use $P c 1$ and $P c 2$ as the SW input time series.

The local scaling characteristics of the principal components are described in the same way as the other SW parameters. The time interval under study was divided into two subsets. The first one (part A in Fig. 5) from 1 January to 14 March 2001 was used for ANN training while the second one (part B in Fig. 5) from 15 March to 28 July 2001 represented an independent set for prediction, not included in the ANN training process. The influence of inclusion of the local Hölder exponents on the ANN performance was tested for a set of values of history $T$ and window length $W$, while $T=W$. In all cases analysed here, a feedback consisting of past $T$ values of $D_{s t}$ index was set. Figure 6 shows the dependence of correlation coefficient $\rho$ (Eq. 6) in three different cases: (1) Hölder exponents $\alpha$ are not considered as input at all - only $P c 1, P c 2$ and the $D_{s t}$ feedback with history $T$ (indicated by a continuous line) are considered; (2) Hölder exponents of $P c 1$ and $P c 2$ vectors are added as input (marked by “*”); (3) as in case (2), but Hölder exponents describing the local scaling properties of past $D_{s t}$ values are also added as an extra input (depicted by "o"). The effect of the inclusion of Hölder exponents is evident mainly in the superior performance of ANNs in case 3. The correlation coeficient $\rho$ achieves its maximum $\rho_{\max }=0.99$ at $W=T=2 \mathrm{~h}$ and decreases with increasing $T$ and $W$. At the same time, ANN performance is practically unchanged in cases 1 and 2 when $T$ and $W$ increase. We mention that without the $D_{s t}$ feedback, $\rho$ slowly increases with $T$ (Jankovičová et al., 2002). As it can be seen, the consideration of scaling properties of $P c 1$ and $P c 2 \mathrm{SW}$ data enhances the performance level of ANN a little, but a real improvement is achieved when the singularity or regularity properties of geomagnetic fluctuations are taken into account as well (case 3). It seems to confirm our expectation that the information on local scaling properies of signals added to the input layer allows one to learn input-output relations better by accounting for changing activity levels more effectively. The analysis of $R M S E$ (Eq. 5) leads to the same conclusion. For demonstration purposes, $1 \mathrm{~h}$ ahead predictions of an intense geomagnetic storm are shown in Fig. 7a. Two methods are compared (Figs. 7b and 7c): case 1 , as defined above, when the $D_{s t}$ index is predicted without Hölder exponents and case 3, with the information on $\alpha \mathrm{s}\left(P c 1, P c 2\right.$ and $\left.D_{s t}\right)$ added to the input layer (the cases 1 and 2 are similar). It is easy to recognize that the method using $\alpha$ s (case 3) allows one to predict almost all the variances in the data with $\rho=0.99$ and $R M S E=2 \mathrm{nT}$ for $T=W=2 \mathrm{~h}$. At the same time $\rho=0.93, R M S E=7 \mathrm{nT}$ for $T=W=2 \mathrm{~h}$ (Fig. 6 in the case without Hölder exponents). In comparison, Wu and Lundstedt (1996) have exploited Elman recurrent ANNs to predict the $D_{s t}$ index $1 \mathrm{~h}$ ahead only from SW data. They achieved $\rho=0.91$ and
$R M S E=16 \mathrm{nT}$.

\section{Conclusions}

We presented a prediction technique which uses the extra information on local scaling exponents to improve the performance of a layered ANN with feedback.

It was demonstrated that the Hölder exponents $\alpha$ are time dependent and change from point to point, exhibiting large deviations from the mean value $\bar{\alpha}=1$, mainly during enhanced activity levels of fluctuations. A peculiar interplay between regularity/irregularity features (described by $\alpha$ ) and amplitude characteristics of disturbances was found and demonstrated on examples of SW and geomagnetic data. ANN performance was significantly improved by adding the Hölder exponent time series of corresponding SW and geomagnetic past data to the input layer, yielding the least $R M S E$ error of $2 \mathrm{nT}$ for short history $T=2 \mathrm{~h}$ and window length $W=2 \mathrm{~h}$. The results obtained without Hölder exponents were the worst $(\rho \sim 0.93, R M S E \sim 7 \mathrm{nT})$. Only a small improvement, if any, was achieved when only the Hölder exponents of SW $P c 1$ and $P c 2$ were added $(\rho \sim$ $0.94, R M S E \sim 6 \mathrm{nT})$. It means that to understand and to model the magnetospheric response better, in addition to SW input and geomagnetic history (feedback), the scaling and irregularity/regularity features of magnetospheric fluctuations should also be taken into account. This is not an unexpected result, however, since recent nonlinear theories on SWMC or magnetotail dynamics involve or predict the appearance of scalings, irregularities (singularities) and turbulence (Galeev et al., 1986; Chang, 1999; Chapman et al., 1999; Klimas et al., 2000). To fully exploit this approach on an experimental basis, further investigations of scalings and singularity features of fluctuations in different inner and outer regions of the magnetosphere will be necessary.

Acknowledgements. The authors wish to acknowledge valuable discussions with P. Kovács, D. Vassiliadis and N. Watkins. $D_{s t}$ index from WDC Kyoto are gratefully acknowledged. We are grateful to N. Ness (Bartol Research Institute) and D.J. McComas (Los Alamos National Laboratory) for making the ACE data available. This work was supported by VEGA grant 2/6040.

\section{References}

Antoni, V., Carbone, V., Cavazzana, R., Regnoli, G., Vianello, N., Spada, E., Fattorini, L., Martines, E., Serianni, G., Spolaore, M., Tramontin, L., and Veltri, P.: Transport processes in reversedfield-pinch plasmas: inconsistency with the self-organized criticality paradigm, Phys. Rev. Lett., 87, 045001-1-045001-4, 2001

Bargatze, L. F., Baker, D. N., McPherron R. L. and Hones, E. W. Magnetospheric response to the IMF: substorms, J. Geophys. Res., 90, 6387, 1985.

Blanchard, G. T. and McPherron, R. L.: A bimodal response function relating the solar wind electric field to the AL index, in "Artificial Intelligence Applications in Solar Terrestrial Physics", Joselyn, J., Lundstedt, H., and Trolinger, J. (eds.), Boulder, NOAA, 153-158, 1994. 
Borovsky, J. E., Elphic, R. C., Funsten, H. O., and Thomsen, M. F.: The Earth's plasma sheet as a laboratory for flow turbulence in high-beta MHD, J. Plasma Phys., 57, 1-34, 1997.

Bruno, R., Bavassano, B., Pietropaolo, E., Carbone, V., and Veltri, P.: Effects of intermittency on interplanetary velocity and magnetic field fluctuations anisotropy, Geophys. Res. Lett., 26, 3185-3188, 1999.

Burlaga, L. F.: Intermittent turbulence in the solar wind, J. Geophys. Res., 96, 5847-5851, 1991.

Carbone, V.: Scaling exponents of the velocity structure functions in the interplanetary medium, Ann. Geophysicae, 12, 585-590, 1994.

Chang, T.: Self-organized criticality, multi-fractal spectra, sporadic localized reconnections and intermittent turbulence in the magnetotail, Phys. Plasmas, 6, 4137-4145, 1999.

Chapman, S. C., Watkins, N. W., Dendy, R. O., Helander, P., and Rowlands, G.: A simple avalanche model as an analogue for magnetospheric activity, Geophys. Res. Lett., 25, 2397-2400, 1998.

Chapman, S. C., Dendy, R. O., and Rowlands, G.: A sandpile model with dual scaling regimes for laboratory, space and astrophysical plasmas, Phys. Plasmas, 6, 4169, 1999.

Consolini, G., Marcucci, M. F., and Candidi, M.: Multifractal structure of auroral electrojet index data, Phys. Rev. Lett., 76, 40824085, 1996.

Consolini, G., and De Michelis, P.: Non-Gaussian distribution function of $A E$-index fluctuations: Evidence for time intermittency, Geophys. Res. Lett., 25, 4087-4090, 1998.

Consolini, G., and Lui, A. T. Y.: Sign-singularity analysis of current disruption, Geophys. Res. Lett., 26, 1673-1676, 1999.

Freeman, M. P., Watkins, N. W., and Riley, D. J.: Power law burst and inter-burst interval distributions in the solar wind: turbulence or dissipative SOC?, Phys. Rev. E, 62, 8794-8797, 2000.

Galeev, A. A., Kuznetsova, M. M., and Zeleny, L. M.: Magnetopause stability threshold for patchy reconnection, Space Sci. Rev., 44, 1-41, 1986.

Gnanadesikan, R.: Methods for statistical data analysis of multivariate observations, John Wiley and Sons, Inc., New York, 1977.

Gonzalez, W. D. and Tsurutani, B. T.: Criteria of interplanetary parameters causing intense magnetic storms $\left(D_{s t}<-100 \mathrm{nT}\right)$, Planet. Space Sci., 35, 1101-1109, 1987.

Guiheneuf, B., Jaffard, S. and Véhel, J. L.: Two results concerning Chirps and 2-microlocal exponents prescription, Appl. Comp. Harm. Anal., 5, 487-492, 1998.

Halsey, T. C., Kadanoff, J. M. H., Procaccia, L. P., and Shraiman, B. I.: Fractal measures and their singularities: the characterization of strange sets, Phys. Rev. A, 33, 1141, 1986.

Hernandez, J. V., Tajima, T., and Horton, W.: Neural net forecasting for geomagnetic activity, Geophys. Res. Lett., 20, 23, 2707, 1993.

Iyemori, T., Maeda, H., and Kamei, T.: Impulse response of geomagnetic indices to interplanetary magnetic field, J. Geomagn. Geoelectr., 6, 577, 1979.

Jaffard, S. and Meyer, Y.: Wavelet methods for pointwise regularity and local oscillations of functions, Memoirs of the A.M.S., 123, 587, 1996.

Jankovičová, D., Dolinský, P., Valach, F., and Vörös, Z.: Neural network based nonlinear determination of the $A E$ index, Contr.
Geophys. \& Geodesy, 31, 343-346, 2001.

Jankovičová, D., Dolinský, P., Valach, F., and Vörös, Z.: Neural network based nonlinear prediction of magnetic storms, J. Atmosph. Solar-Terr. Phys., in press, 2002.

Klimas, A. J., Valdivia, J. A., Vassiliadis, D., Baker, D. N., Hesse, and Takalo, J.: Self-organized criticality in the substorm phenomenon and its relation to localized reconnection in the magnetospheric plasma sheet, J. Geophys. Res., 105, 18765-18780, 2000.

Kovács, P., Carbone, V., and Vörös, Z.: Wavelet-based filtering of intermittent events from geomagnetic time series, Planet. Space Sci., 49, 1219-1231, 2001.

Kröse, B. and Smagt, P.: An introduction to neural networks, The University of Amsterdam, 1996.

Mallat, S. G. and Hwang, W. L.: Singularity detection and processing with wavelets, IEEE Trans. Inform. Theory, 38(2), 617-643, 1992.

Marsch, E., Tu, C. Y., and Rosenbauer, H.: Multifractal scaling of the kinetic energy flux in solar wind turbulence, Ann. Geophysicae, 14, 259-269, 1996.

McPherron, R. L., Baker, D. N., Bargatze, L. F., Clauer, C. R., and Holzer, R. E.: IMF control of geomagnetic activity, Adv. Space Res., 8, 71, 1988.

Munsami, V.: Determination of the effects of substorms on the storm-time ring current using neural networks, J. Geophys. Res., 105, $27833-27840,2000$.

Muzy, J. F., Bacry, E., and Arneodo, A.: Multifractal formalism revisited with wavelets, Int. J. Bifurc. Chaos, 4, 245-302, 1994.

Price, C. P., Prichard, D., and Bischoff, J. E.: Nonlinear input/output analysis of the auroral electrojet index, J. Geophys. Res., 99, 7, $13277,1994$.

Riedi, R. H. and Véhel, J. L.: Multifractal properties of TCP traffic:a numerical study, INRIA Res. Rep., 3129, 1997.

Reyment, R. A. and Jöreskog, K. G.: Applied factor analysis in the natural sciences, Cambridge University Press, 1996.

Rumelhart, D. E., Hinton, G., and Williams, R.: Learning representations by back-propagating errors, Nature, 323, 533, 1986

Tu, C. Y., Marsch, E., and Rosenbauer, H.: An extended structurefunction model and its application to the analysis of solar wind intermittency properties, Ann. Geophysicae, 14, 270-285, 1996.

Vassiliadis, D., Klimas, A. J., Baker, D. N. and Roberts, D. A.: A description of the solar wind-magnetosphere coupling based on nonlinear filters, J. Geophys. Res., 100, 3495-3512, 1995.

Véhel, J. L., and Vojak, R.: Multifractal analysis of choquet capacities: preliminary results, Adv. Appl. Math., 20, 1-43, 1998.

Vörös, Z.: On multifractality of high-latitude geomagnetic fluctuations, Ann. Geophysicae, 18, 1273-1282, 2000.

Watkins, N. W., Freeman, M. P., Chapman, S. C. and Dendy, R. O.: Testing the SOC hypothesis for the magnetosphere, J. Atmosph. Sol. Terr. Phys., 63, 1435-1445, 2001.

Weigel, R. S., Horton, W., Tajima, T., and Detman, T.: Forecasting Auroral electrojet activity from solar wind input with neural networks, Geophys. Res. Lett., 26, 1353-1356, 1999.

Weigel, R. S.: Prediction and modeling of magnetospheric substorms, Thesis, 2000.

Wu, J. G., and Lundstedt, H.: Prediction of geomagnetic storms from solar wind data using Elman recurrent neural networks, Geophys. Res. Lett., 23, 319-322, 1996. 